\title{
28 Research Square \\ Epidemic analysis of COVID-19 in China after Wuhan was restricted
}

anfeng yu

Wuhan University Renmin Hospital

zhiwei wang ( $\nabla$ wangzhiwei@whu.edu.cn )

Wuhan University Renmin Hospital https://orcid.org/0000-0001-5643-9344

wei ren

Wuhan University Renmin Hospital

zhiyong wu

Wuhan University Renmin Hospital

zhipeng hu

Wuhan University Renmin Hospital

luocheng li

Wuhan University Renmin Hospital

yongle ruan

Wuhan University Renmin Hospital

rui hu

Wuhan University Renmin Hospital

feng shi

Wuhan University Renmin Hospital

\section{Research}

Keywords: Coronavirus Disease 2019, Severe Acute Respiratory Syndrome Coronavirus 2, Wuhan, Restrict, Change of epidemic situation

Posted Date: February 24th, 2020

DOI: https://doi.org/10.21203/rs.2.24289/v1

License: (c) (1) This work is licensed under a Creative Commons Attribution 4.0 International License. Read Full License 


\section{Abstract}

\section{Background}

In December 2019, Coronavirus Disease 2019(COVID-19) caused by Severe Acute Respiratory Syndrome Coronavirus 2 (SARS-CoV-2) infection appeared in Wuhan, Hubei Province, China. The disease is highly infectious. Wuhan, Hubei Province decided restrict personnel movement on January 23.We analyze relevant data to show the situation of the COVID-19 epidemic in China.

\section{Methods}

The data was classified according to Hubei group, non-Hubei group, Hong Kong, Macao and Taiwan group, and Chinese Mainland group, and analyze the current situation and trend of the epidemic.

\section{Results}

There was an explosive growth in the early stage of the epidemic. The epidemic situation began to improve in about two weeks after Wuhan was restricted,and the situation in non-Hubei was significantly better than that in Hubei.

\section{Conclusion}

The blockade of Wuhan was a correct decision, cut off the outflow of tinfection sources, and the epidemic situation in all places turned around after the incubation period.

\section{Introduction}

In December 2019, Wuhan, Hubei Province, China, had some pneumonia of unknown causes, all of which were related to the local South China seafood market ${ }^{[1]}$. According to the investigation of Chinese Center for Disease Control and Prevention(CCDC), the pathogen of pneumonia is a new type of coronavirus named Severe Acute Respiratory Syndrome Coronavirus 2 (SARS-CoV-2).

The virus is highly infectious, forming a clustering outbreak in Wuhan in a short time. Since January is around the Chinese Lunar New Year, there are a large number of people returning to their hometown. In order to avoid the further spread of the virus, Wuhan, Hubei Province decided on January 23 to restrict the movement of people in Wuhan,close all foreign transportation hubs in Wuhan. So far, it has been 23 days since Wuhan was closed. We have collected all the information about the COVID-19 epidemic caused by SARS-CoV-2, and made a summary analysis report on the information.

\section{Methods}

Through the collection of COVID-19 epidemic information from January 10 to January 14 by the National Health Committee of the People's Republic of China and the Health Committees of all provinces and 
cities in China.The epidemic information was classified and analyzed according to Hubei group, nonHubei group, Hong Kong, Macao and Taiwan group, and Chinese Mainland group, and analyze the current situation and trend of the epidemic, and compare the differences in severe rate, cure rate and mortality between Hubei and non-Hubei regions.

\section{Statistical analysis}

All the data were expressed as mean \pm standard deviation, and all the statistical analyses were done using the Graphpad Prism 8.0 software. The independent Student's t-test was used to compare the data between two groups. In both cases, differences with $\mathrm{P}<0.05$ were considered statistically significant.

\section{Results}

Since the outbreak of the disease was found in Chinese Mainland until February 4, the number of new patients per day continues to rise. After February 4, there was a downward trend, that is, 13 days after restricting the movement of people in Wuhan, the national epidemic began to improve. In order to help Hubei Province control the epidemic more quickly, so that suspected patients can be treated more quickly, $\square$ A novel coronavirus pneumonia diagnosis and treatment plan(trial version fifth) $\square$ was launched in the National Health Committee of the People's Republic of China in February 12th ${ }^{[3]}$. "Clinical diagnosis" has been added to the classification of diagnosis in Hubei Province, that is,suspected cases with imaging characteristics of pneumonia, but there is no evidence of etiology. As a result, there was an explosive increase in the number of newly diagnosed cases on February 12, and then they recovered to their previous levels and showed a downward trend. In Chinese Mainland, the severe rate of patients with COVID-19 is relatively stable at $19.03 \% \pm 3.48 \%$. The cure rate was on the rise in the first few days when the outbreak did not occur.From January 16 to January 27, the cure rate of patients continued to decline after the outbreak. With the control of epidemic situation and the standardization of diagnosis and treatment, the cure rate has gradually increased to more than $12 \%$. It can be predicted that the cure rate will gradually increase. Although there is no completely effective treatment plan for COVID-19 at present, due to the progress of medical intensive care technology, the mortality rate of patients with COVID-19 in Chinese Mainland is basically stable at $2.48 \% \pm 0.64 \%$. Before the specific treatment plan comes out, the mortality rate will not change much(Fig. 1).

Hubei is the main gathering place of the epidemic, $80 \%$ of the patients with COVID-19 in China are concentrated in Hubei Province at present. Therefore, the epidemic situation in Hubei is similar to that in Chinese Mainland. The change of the epidemic situation in Hubei will affect the overall development of Chinese Mainland(Fig. 2).

The epidemic situation in non-Hubei area is much lighter, Since February 3, the number of new patients per day has continued to decline significantly, that is to say, 12 days after the restriction of Wuhan City, there has been a significant improvement. It can be preliminarily estimated that the number of new diseases will reach a negative growth in the next week. The severe rate of patients with COVID-19 in non- 
Hubei area has been declining slowly since the beginning of the epidemic. At present, the critical rate is only $7.45 \%$. It is believed that it will continue to decline in the future. The cure rate in non-Hubei areas has been on the rise, and now it has reached $27.80 \%$. In non-Hubei area, patients with COVID-19 have mild illness and low mortality rate. At present, the mortality rate is only $0.69 \%$ (Fig. 3).

Due to the great difference between Hubei and non-Hubei areas, the severe rate, cure rate and mortality of Hubei and non-Hubei areas were compared. It can be found that the severe rate and mortality of nonHubei areas are significantly lower than those of Hubei(Table).

Due to the special geographical location of Hong Kong, Macao and Taiwan and the prevailing policy with the Chinese Mainland, the overall epidemic situation in Hong Kong, Macao and Taiwan is relatively slow to rise, but there is no obvious downward trend at present, and the current new cases are mainly concentrated in Hong Kong.Macao and Taiwan have not seen new patients for about one week in a row(Fig. 4).

\section{Discussion}

The pathogen of COVID-19 is a new type of coronavirus belonging to the Beta Coronavirus genus. Coronavirus group of International Virus Classification Commission named it SARS-CoV-2. Shi Zhengli's team found that the gene sequence of SARS-CoV-2 is up to $96 \%$ homologous to the coronavirus carried by the Chinese chrysanthemum, and it is speculated that the Chinese chrysanthemum may be the natural host for SARS-CoV-2 ${ }^{[2]}$. So far, the main source of infection is COVID-19 patients, and respiratory droplet transmission is the main route of transmission, which can also be transmitted through contact ${ }^{[3]}$. Zhang Yuanzhen's team found that there was no evidence of vertical transmission of the virus from mother to child $^{[4]}$. Zhong Nanshan's team found that SARSCoV-2 was isolated from the stool of COVID-19 patients, but further research is needed on whether the virus can be transmitted through the fecal-oral route.

According to the current epidemiological survey, the median incubation period of the virus after infection is 3-7 days, with a maximum incubation period of 2 weeks. Although there are 24 day incubation period reported, it is still to be studied whether the specific patient is infectious ${ }^{[5]}$. The main symptoms of patients with COVID-19 were fever, dry cough, fatigue, dyspnea and Upper respiratory symptoms such as nasal obstruction and runny nose ${ }^{[6]}$. Wang Weiguo's team also pointed out atypical symptoms, such as diarrhea, headache and dizziness ${ }^{[7]}$.According to the report of Zhongnan Hospital of Wuhan University, typical lung CT shows multiple, patchy, sub segmental or segmental ground glass density shadows in both lungs, at present, the main examination methods are hematology examination, nucleic acid examination and chest CT examination ${ }^{[8]}$.

The results of Wan Yushun's research show that the receptor for the SARS-CoV-2 is AngiotensinConverting Enzyme 2(ACE2). It is speculated that the drugs for ACE2 may be suitable for the SARS-CoV-2, so it can be used as the first choice for disease prevention and treatment. On the other hand, the vaccine based on coronavirus S-protein receptor binding region (RBD) is also worthy of further study ${ }^{[9]}$. 
More than two months have passed since the discovery of the first COVID-19 patient, during this period, China's epidemic experienced an explosive growth. Although the cumulative number of patients has reached 66492, the overall epidemic situation is controllable. The non-Hubei area has improved obviously, and the Hubei area has also shown a trend of improvement. There are five reasons for analysis: 1. Improvement of diagnosis and treatment program;2. Deepening of research on SARS-CoV-2 by scientific researchers;3. Construction of designated hospital;4. Establishment of shelter hospital;5. Efforts of medical staff.There are six reasons for the outbreak: 1 . SRAS-CoV-2 is a new coronavirus, and there is no specific treatment plan at present;2. People didn't pay attention to this at the beginning of the outbreak;3. The SRAS-CoV-2 has a long incubation period of about 2 weeks, which is consistent with the previous data. Two weeks after the closure of Wuhan, the epidemic situation in non Hubei area improved significantly;4. Wuhan is an important transportation hub in China, with developed transportation and convenient access;5. January 2019 is the Chinese Lunar New Year, a large number of people return home to visit their families; 6 . Wuhan is the city with the largest number of college students in China. A large number of students returned home during the winter vacation in January.

In the face of this epidemic, we have exposed a lot of deficiencies, which is manifested in the fact that the important problems in the process of the epidemic, such as the variation characteristics, transmission mode and traceability of the SRAS-CoV-2, have not been completely solved. We also need to work hard to meet the challenges of more new pathogens in the future.

\section{Conclusion}

Restricting the movement of people in Wuhan on January 23, 2020 was a correct decision, effectively curbing the greater development of the epidemic, although more than 20 days have passed since the outbreak began. Wuhan is currently found to be the source of the epidemic. After restricting the movement of people in Wuhan, the source of infection was confined to Wuhan as much as possible, unable to move domestically, reducing the risk of infection in other domestic provinces and cities.

Although the movement of some people due to various reasons in the early stage of the epidemic also caused the outbreak in the country, the epidemic situation in various places improved significantly after 2 weeks (latency period) after the city was closed. The severity and mortality of non-Hubei regions were also significantly lower than those of Hubei.

\section{Abbreviations}

COVID-19: Coronavirus Disease 2019

SARS-CoV-2: Severe Acute Respiratory Syndrome Coronavirus 2

CCDC: Chinese Center for Disease Control and Prevention

ACE2: Angiotensin-Converting Enzyme 2 
RBD: S-protein receptor binding

\section{Declarations}

1.Ethics approval and consent to participate: Not applicable

2.Consent for publication: Not applicable

3.Availability of data and materials: The datasets used and analysed during the current study are available from the corresponding author on reasonable request.

4.Competing interests: The authors declare that they have no competing interests.

5.Funding: This work is funded by the National Natural Science Foundation of China (Grant No.81570428), Key Support Project of Health Commission of Hubei Province (Grant No.WJ2019Z012),Guiding Fund of Renmin Hospital of Wuhan University (Grant No.RMYD2018Z07).

6.Authors' contributions:Anfeng Yu designed the study and contributed to manuscript writing,Zhiwei Wang participated in the design of the study. Wei Ren,Zhiyong Wu,Zhipeng Hu,Luocheng Li,Yongle Ruan,Rui Hu, Feng Shi contributed to data collection.

8.Acknowledgements: Thank you to the National Health Committee of the People's Republic of China, the Health Committees of the provinces and cities, as well as the medical staff and scientific researchers who are struggling to provide data. Finally pay our highest respect to our lovely and strong healthcare workers. Keep in mind the medical staff and other related personnel who were sacrificed during the epidemic, such as Dr. Li Wenliang.

\section{References}

1. Huang $C$, Wang $Y, L i X$, et al. Clinical features of patients infected with 2019 novel coronavirus in Wuhan, China [published online ahead of print, 2020 Jan 24] Lancet. 2020.doi:10.1016/S01406736(20)30183-5

2. Zhou $P$, Yang $X$, Wang $X$, et al. Discovery of a novel coronavirus associated with the recent pneumonia outbreak in humans and its potential bat origin[EB/OL]. [2020冈01ه30].https://www.biorxiv.org/ content/10.1101/2020.01.22.914952v1.full.pdf

3. General Office of National Health Committee. Office of State Administration of Traditional Chinese Medicine. Notice on the issuance of a programme for the diagnosis and treatment of novel coronavirus (2019-nCoV) infected pneumonia (Trial Version 5). 2020. http://bgs.satcm.gov.cn/

4. Chen $\mathrm{Hj}$,Guo Jj,Wang C,et al. Clinical characteristics and intrauterine vertical transmission potential of COVID-19 infection in nine pregnant women: a retrospective review of medical records.Lancet.https://doi.org/10.1016/S0140-6736(20)30360-3 
5. Zhou P, Yang XL, Wang XG, Hu B, Zhang L, Zhang W, et al. A pneumonia outbreak associated with a new coronavirus of probable bat origin. Nature. 2020. https://doi.org/10.1038/s41586-020-2012-7.

6. Zhu N, Zhang D, Wang W, Li X, Yang B, Song J, et al. A novel coronavirus from patients with pneumonia in China, 2019. N Engl J Med.2020.https:// doi.org/10.1056/NEJMoa2001017.

7. Wang W, Hu H, Song L, Gong X, Qu Y, Lu Z. Image of pulmonary and diagnosis of atypicalnovel coronavirus (2019-nCoV) infected pneumonia: case series of 14 patients. Yixue Xinzhi.2020;30(1):79 [Article in China].

8. Jin YH, Cai L, Cheng ZS, et al. A rapid advice guideline for the diagnosis and treatment of 2019 novel coronavirus (2019-nCoV) infected pneumonia (standard version). Mil Med Res. 2020;7(1):4. doi:10.1186/s40779-020-0233-6

9. Wan Y, Shang J, Graham R, Baric RS, Li F. Receptor recognition by novel coronavirus from Wuhan: An analysis based on decade-long structural studies of SARS [published online ahead of print, $2020 \mathrm{Jan}$ 29]. J Virol.2020.doi:10.1128/JVI.00127

\section{Table}

Comparison of severe rate,cure rate and mortality between Hubei and non-Hubei regions

\begin{tabular}{cccc}
\hline & Hubei & non-Hubei & P value \\
\hline Severe rate(\%) & $22.35 \pm 3.70$ & $12.49 \pm 8.25$ & $<0.0001 *$ \\
Cure rate(\%) & $8.82 \pm 8.31$ & $7.34 \pm 8.18$ & 0.4967 \\
Mortality(\%) & $3.22 \pm 0.89$ & $0.69 \pm 0.85$ & $<0.0001 *$ \\
\hline
\end{tabular}

* $P<0.0001$

Figures 
A

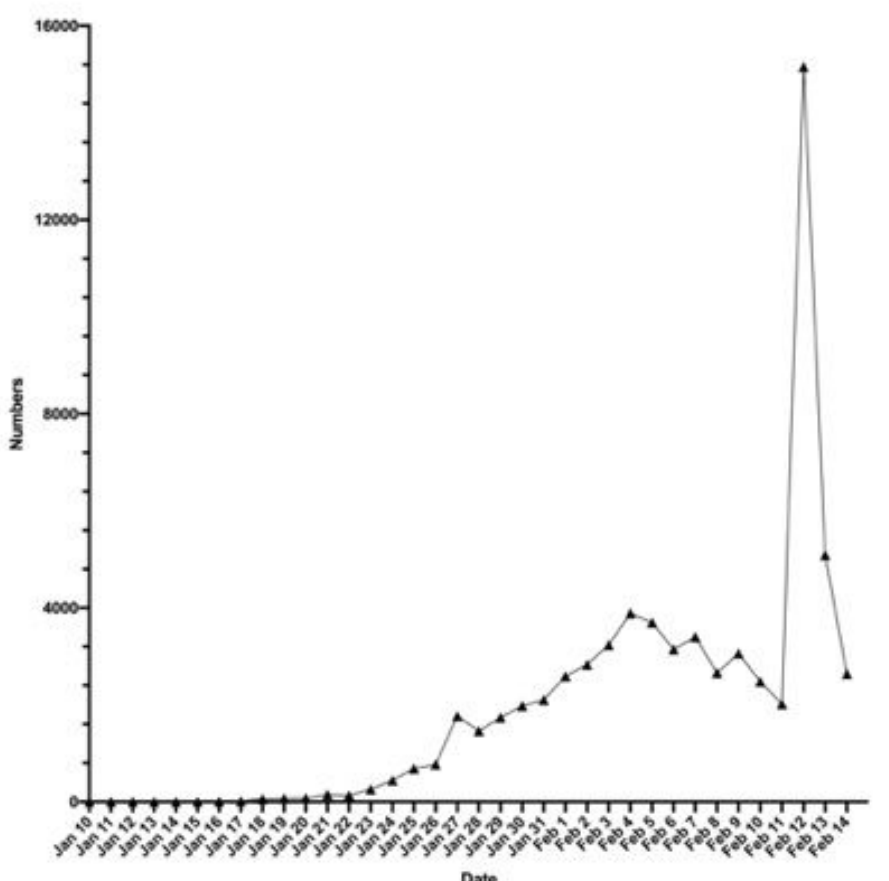

$\mathrm{C}$

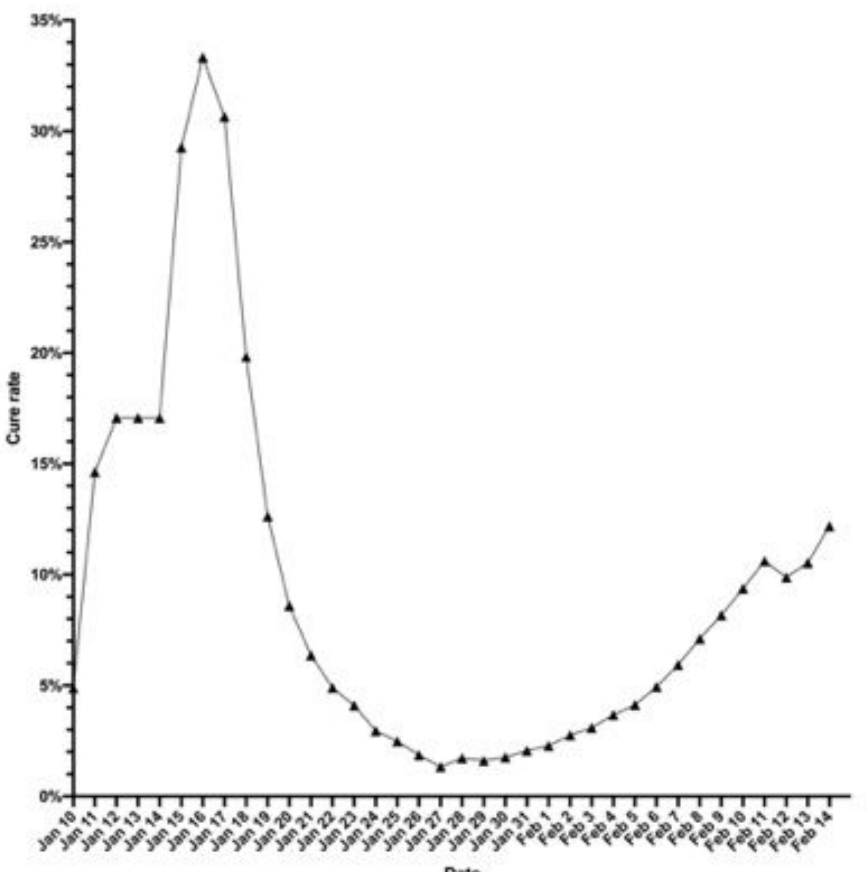

B
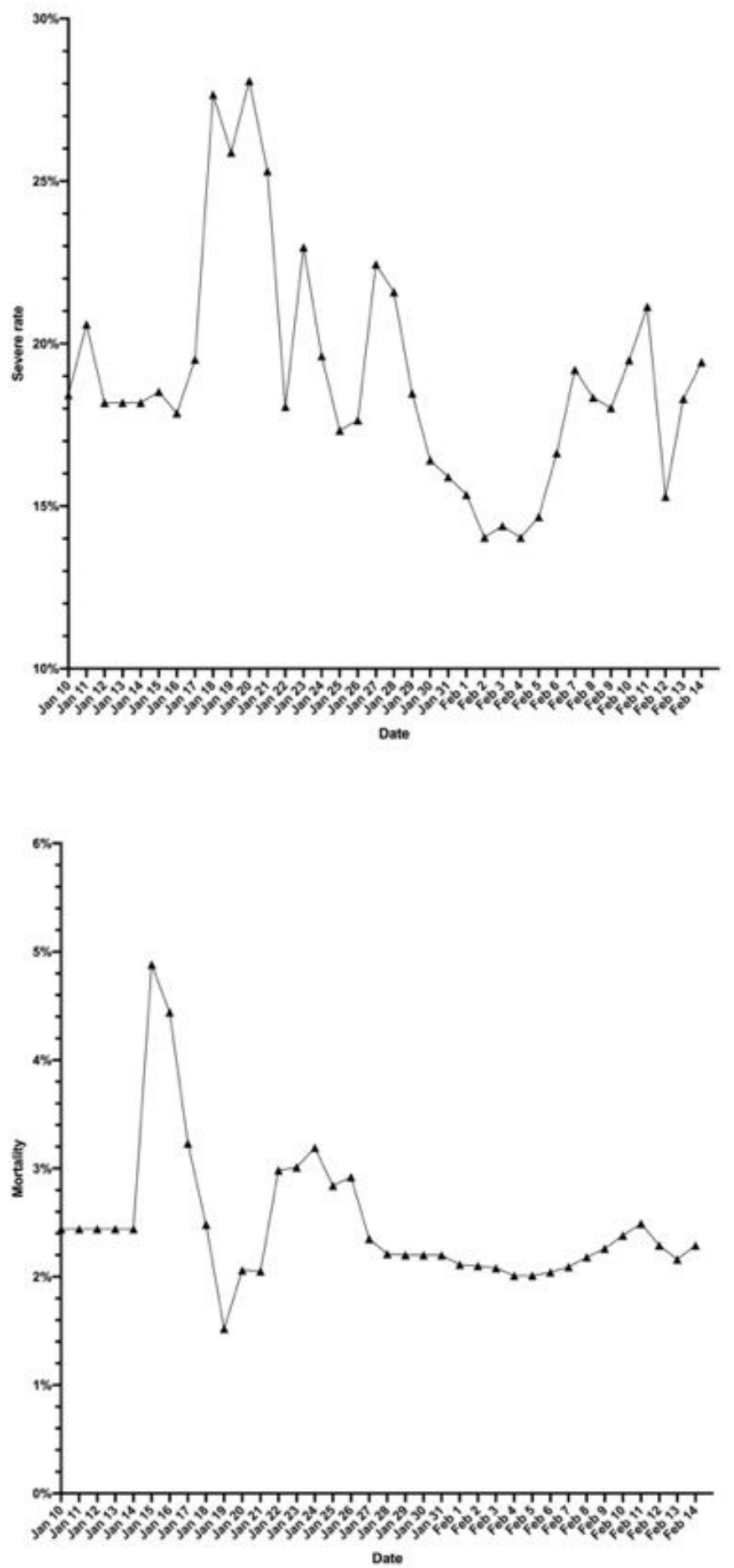

Figure 1

Epidemic situation in Chinese Mainland A, Newly confirmed patients with COVID-19 in Chinese Mainland B, Severe rate of patients with COVID-19 in Chinese Mainland C, Cure rate of patients with COVID-19 in Chinese Mainland D, Mortality of patients with COVID-19 in Chinese Mainland 
A

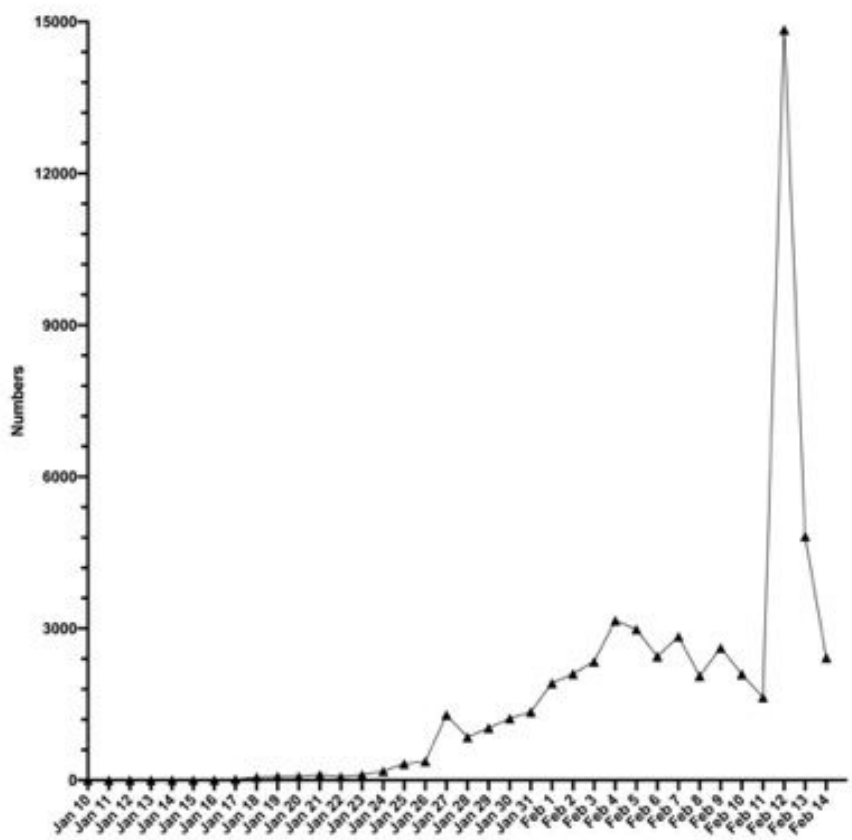

Date

$\mathrm{C}$

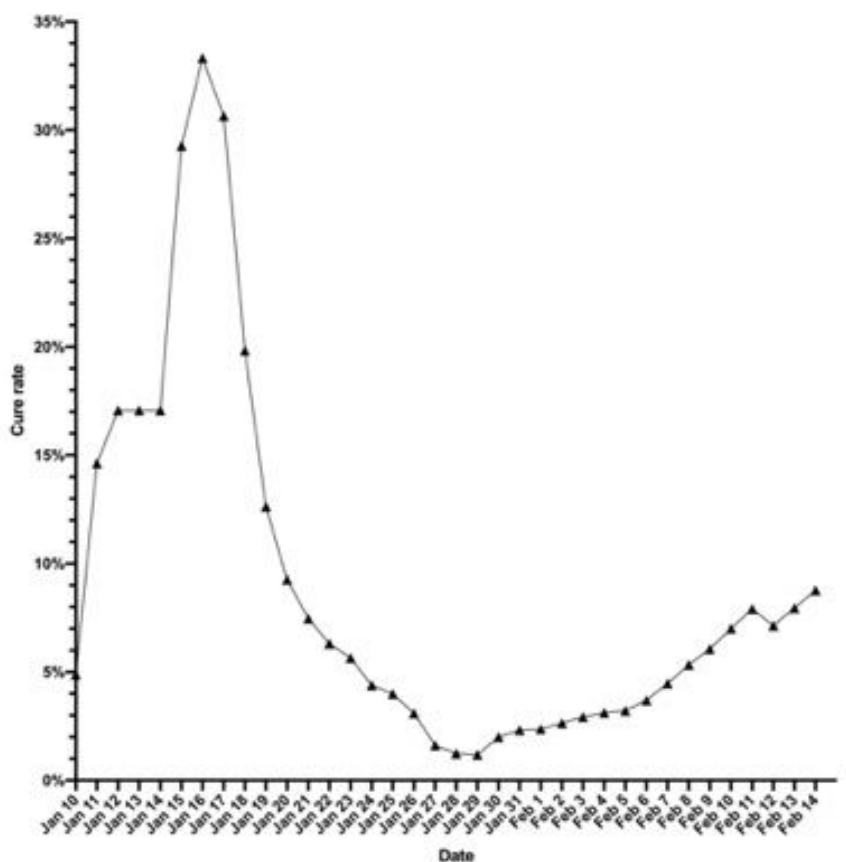

B

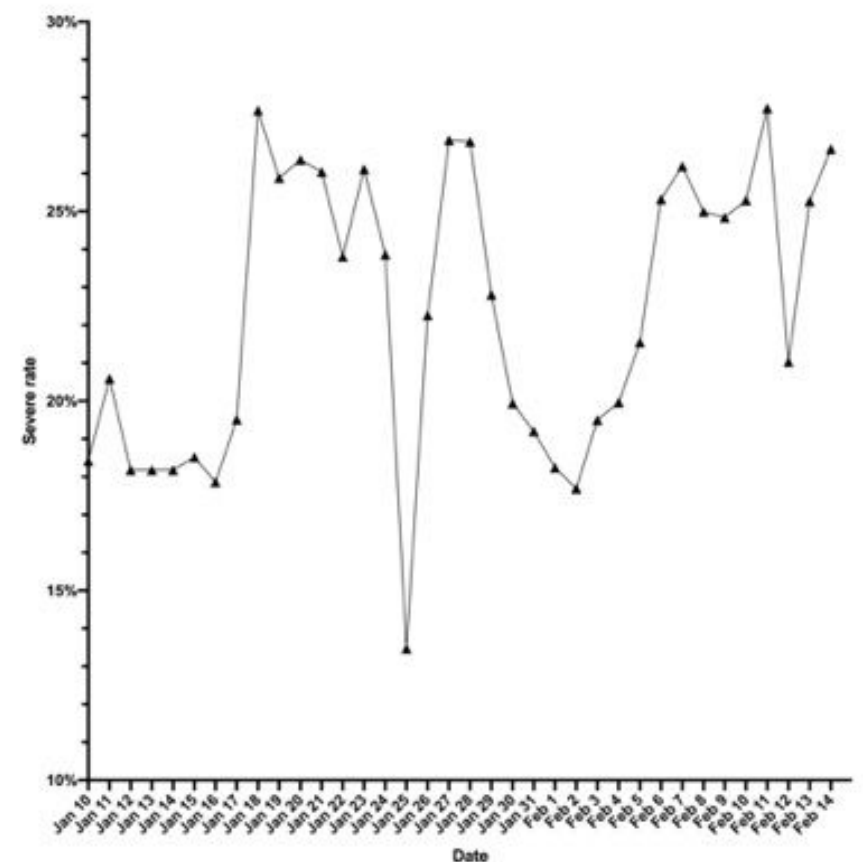

D

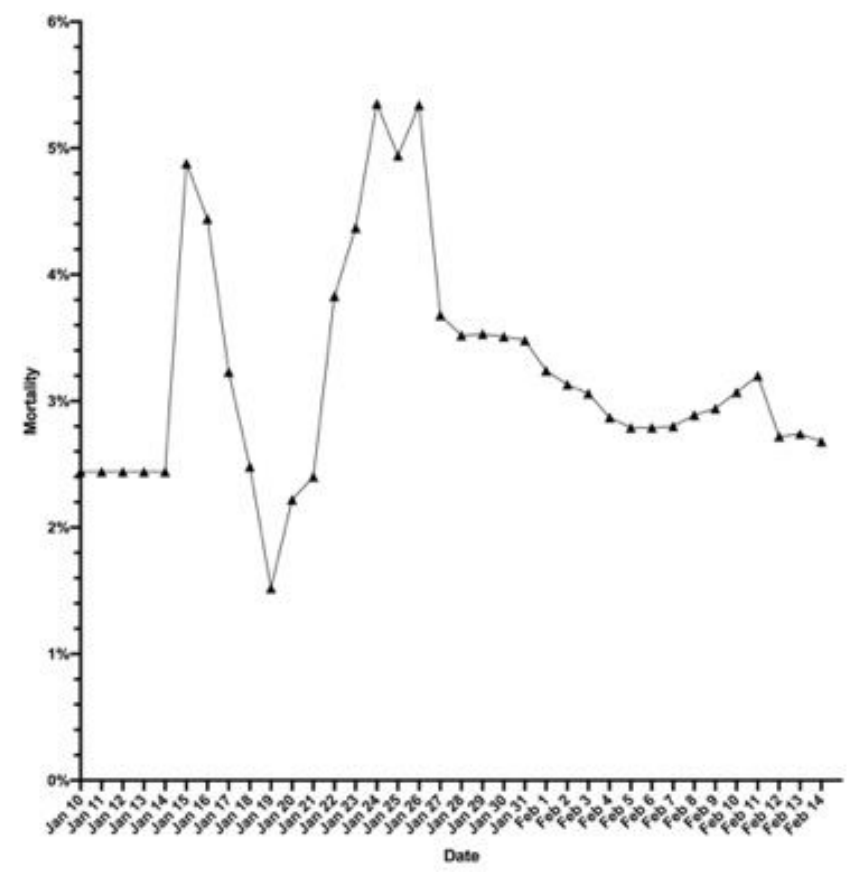

Figure 2

Epidemic situation in Hubei A, Newly confirmed patients with COVID-19 in Hubei B, Severe rate of patients with COVID-19 in Hubei C, Cure rate of patients with COVID-19 in Hubei D, Mortality of patients with COVID-19 in Hubei 
A

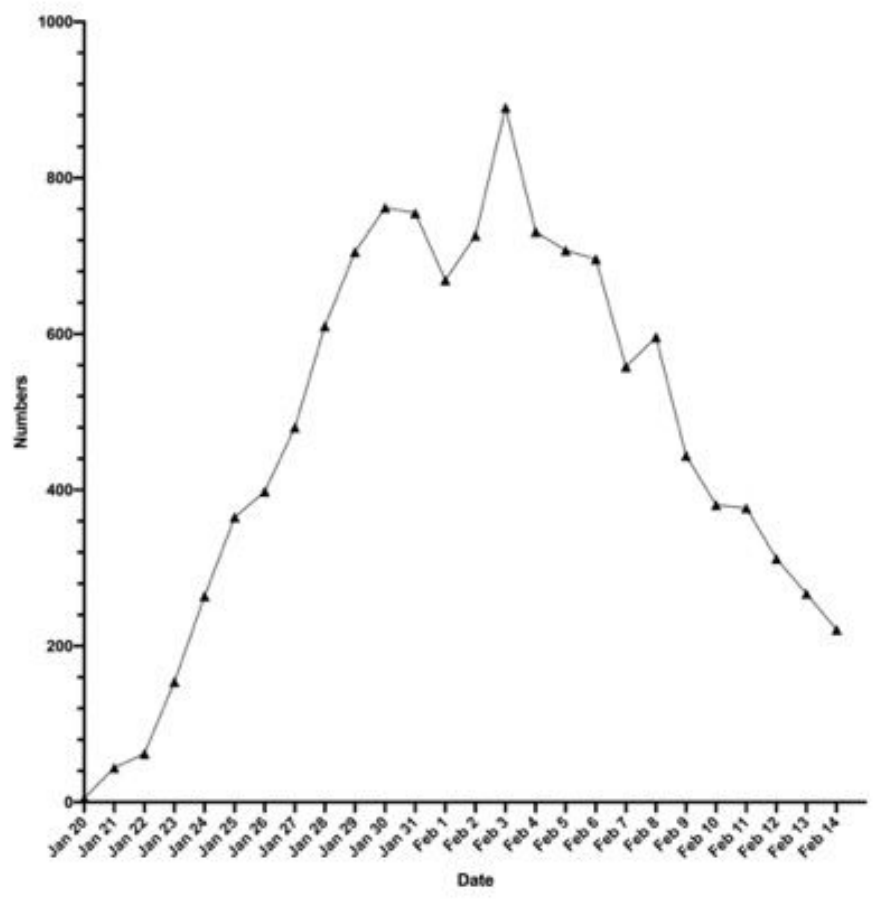

$\mathrm{C}$

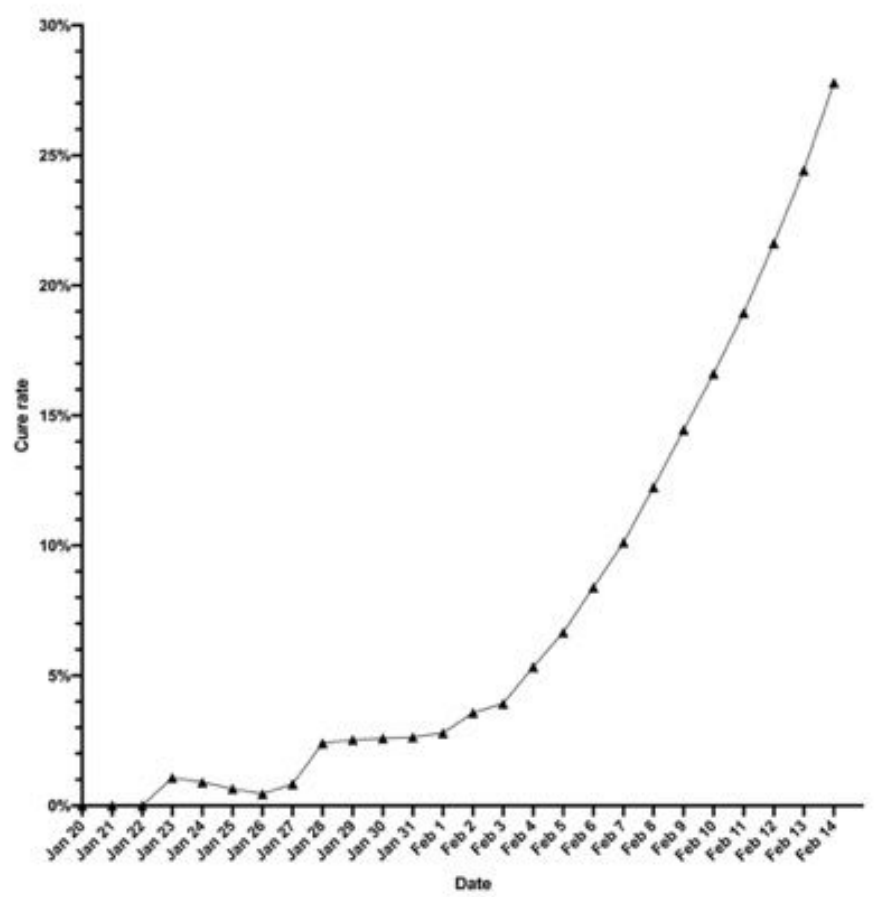

B

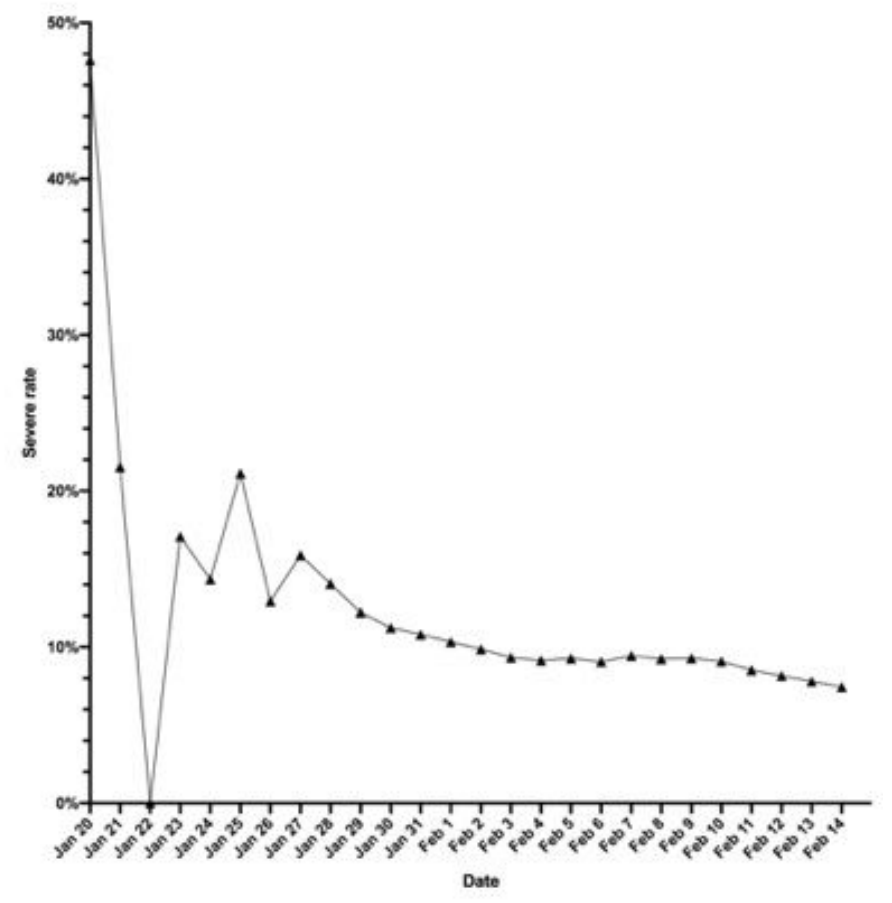

D

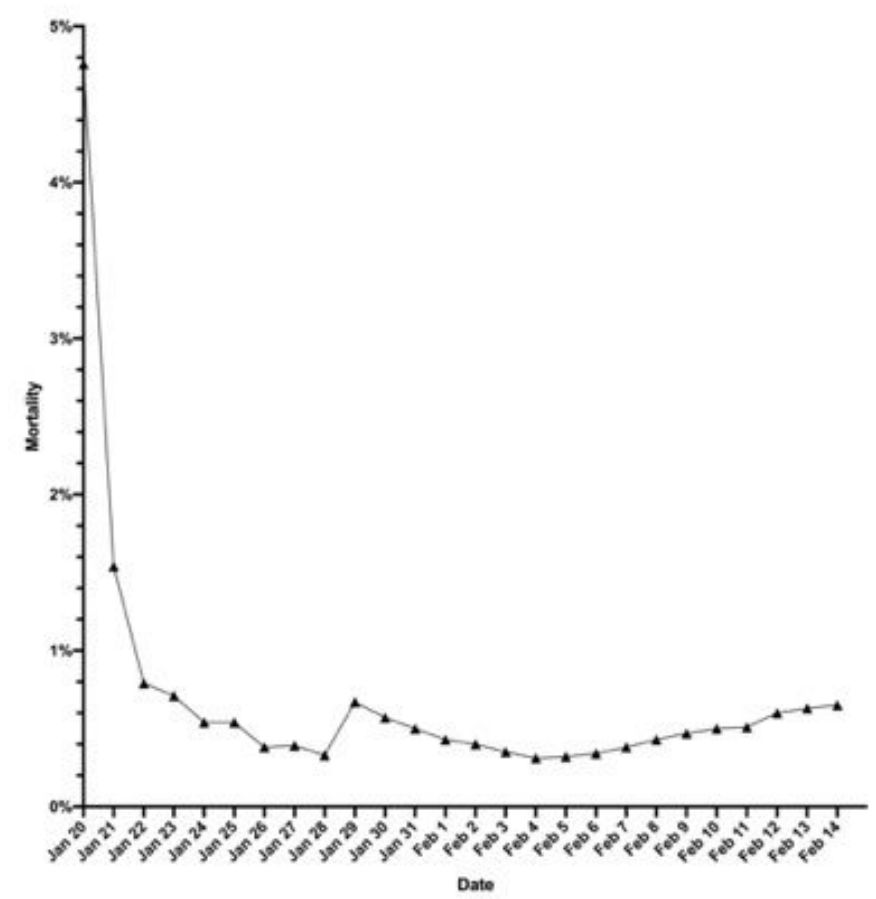

Figure 3

Epidemic situation in non-Hubei areas A, Newly confirmed patients with COVID-19 in non-Hubei areas B, Severe rate of patients with COVID-19 in non-Hubei areas C, Cure rate of patients with COVID-19 in nonHubei areas D, Mortality of patients with COVID-19 in non-Hubei areas 
A

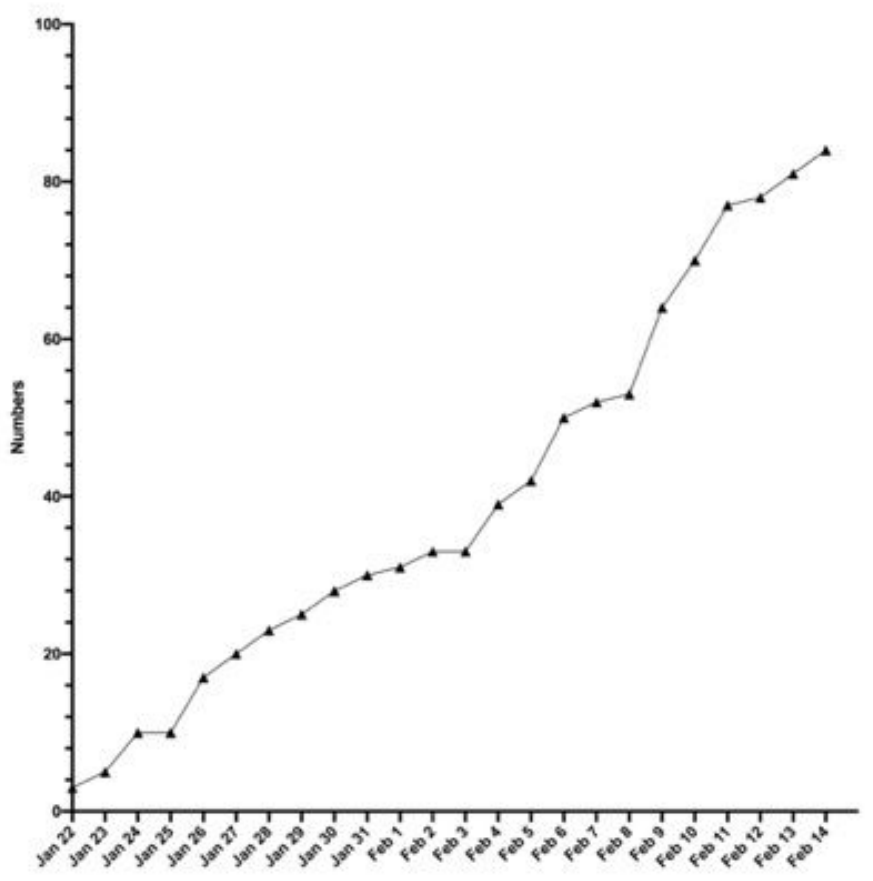

Date

$\mathrm{C}$

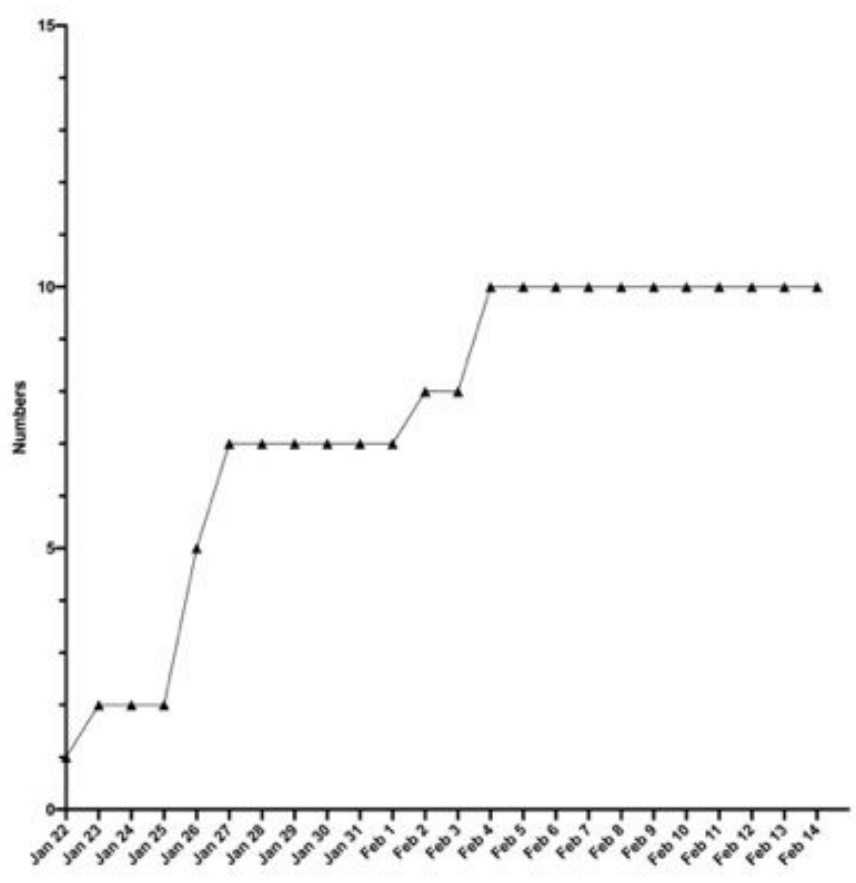

Dato
B

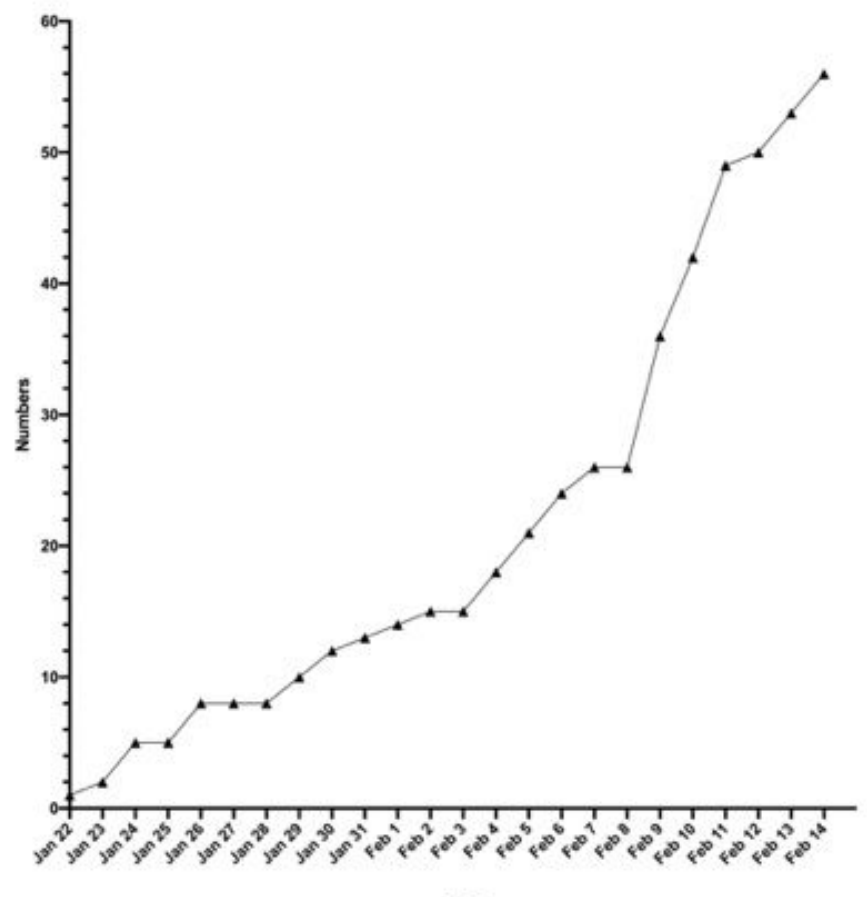

Dato

$\mathrm{D}$

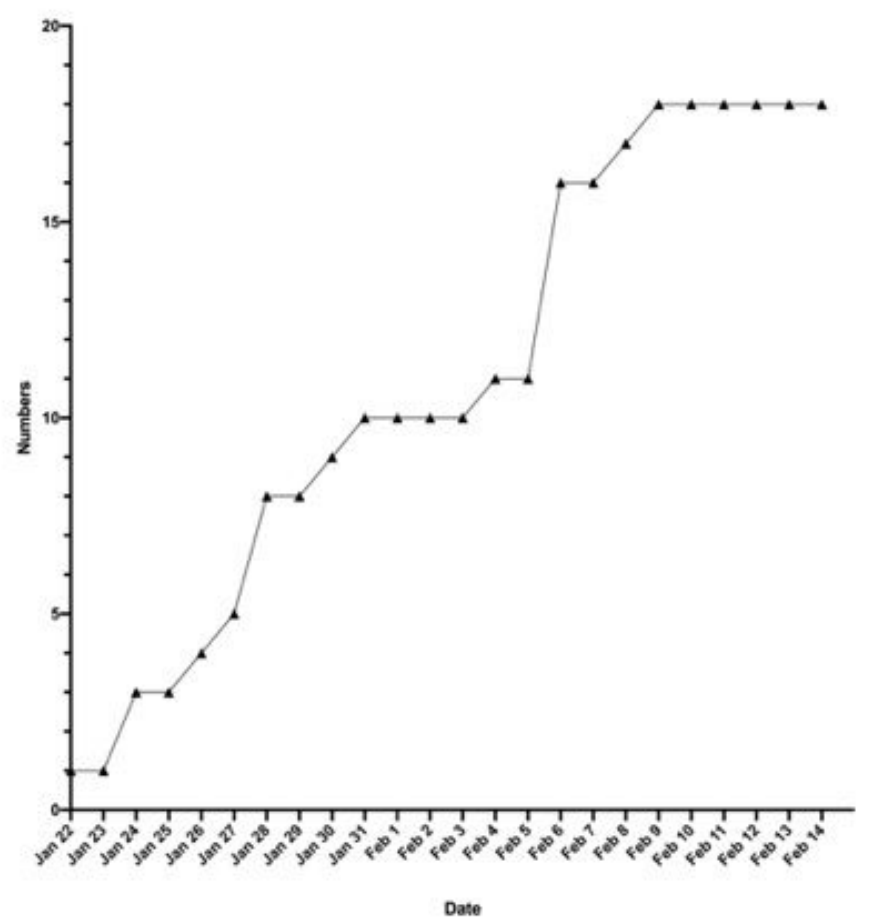

\section{Figure 4}

Epidemic situation in Hong Kong, Macao and Taiwan A, Cumulative growth trend of COVID-19 patients in Hong Kong, Macao and Taiwan B, Cumulative confirmed patients with COVID-19 in Hong Kong C, Cumulative confirmed patients with COVID-19 in Macao D, Cumulative confirmed patients with COVID-19 in Taiwan 